\title{
The biomechanical differences of rotational acetabular osteotomy, Chiari osteotomy and shelf procedure in developmental dysplasia of hip
}

\author{
Ming $\mathrm{Fu}^{1{ }^{* \dagger}}$, Shanshan Xiang ${ }^{2+}$, Zhiqi Zhang ${ }^{1 *}$, Guangxin Huang ${ }^{1}$, Jin Liu', Xin Duan ${ }^{1}$, Zibo Yang ${ }^{1}$, Peihui Wu \\ and Weiming Liao'
}

\begin{abstract}
Background: Rotational acetabular osteotomy (RAO), Chiari osteotomy and shelf procedure are important treatments to delay the progression of osteoarthritis in developmental dysplasia of hip (DDH) patients, but their biomechanical differences are still unknown. This study was to evaluate the different biomechanical changes of hip joint after these three surgeries.

Methods: Sixteen DDH models of 8 human cadaver specimens were reconstructed, and treated by different surgeries, and then strain around femoral head was evaluated by strain gauges.

Results: Hip strain value of DDH model was decreased after treated by shelf procedure (Pleft $=0.016$ and Pright $=0.021$ ) and rotational acetabular osteotomy $(P=0.004)$, but not in Chiari osteotomy $(P=0.856)$. Moreover, the improved ratio of RAO treatment was better than shelf procedure $(P=0.015)$ and Chiari osteotomy $(P=0.0007)$, and the descendent range of shelf procedure was greater than Chiari osteotomy $(P=0.018)$.
\end{abstract}

Conclusions: From biomechanics points, RAO was more effective in relieving hip joint stress compared with shelf procedure and Chiari osteotomy.

Keywords: Rotational acetabular osteotomy, Chiari osteotomy, Shelf procedure, Hip stress

\section{Background}

Developmental Dysplasia of Hip (DDH) is a common congenital deformity of hip. If appropriate measures are not taken, DDH often develops into secondary hip osteoarthritis (OA) due to the abnormal stress of hip [1-5]. Although total hip arthroplasty (THA) is an effective treatment for late stage OA, its application for young DDH patients is still controversial in consideration of the survival rate of prosthesis [6,7]. It is widely accepted that joint preserving operations such as periacetabular osteotomy should be selected whenever possible [8-12]. Rotational acetabular osteotomy (RAO), Chiari osteotomy and shelf procedure are three different periacetabular osteotomies, which are often used for adolescent

\footnotetext{
*Correspondence: sysumf@126.com; zhzhiqi@163.com

${ }^{\dagger}$ Equal contributors

'Department of Joint Surgery, First Affiliated Hospital, Sun Yat-sen University, Guangzhou 510080, China

Full list of author information is available at the end of the article
}

and adult DDH. All of them could re-adjust the positional relationship of the acetabulum and femoral head to increase the acetabular coverage of the femoral head, restore normal anatomical structures, and then improve the biomechanical properties of hip. Previous studies often focused on the clinical outcomes of these three procedures [13-19], while relatively little knowledge is available related to the postoperative biomechanical changes of hip. Iliescu $\mathrm{N}$ et al. reported that the principal stress on the surface of the acetabulum was decreased by $17.4 \%$ after Chiari osteotomy in a photoelastic investigation, although it was still greater than a normal hip [20]. And it was shown on a computer model that increasing the height of Chiari osteotomy from acetabular rim decreased the load-bearing contact area as well as increased the contact pressure of hip [21]. However, the different biomechanical changes among these three operations have not been reported before.

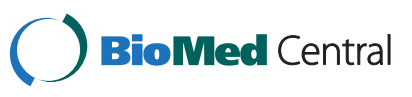

of the article

(c) 2014 Fu et al.; licensee BioMed Central Ltd. This is an Open Access article distributed under the terms of the Creative Commons Attribution License (http://creativecommons.org/licenses/by/2.0), which permits unrestricted use, distribution, and reproduction in any medium, provided the original work is properly credited. 
In this study, RAO, Chiari osteotomy and shelf procedure were performed on sixteen DDH models which were established on eight adult cadaver pelvis specimens. Hip strain was measured before and after these three osteotomies in an attempt to investigate the different biomechanical changes of hip after operations and compare their efficiency in reducing the abnormal stress of hip in DDH.

\section{Methods}

\section{Subjects}

This study was approved by the Medical Ethics Committee of Sun Yat-sen University. Twelve female cadavers who had died at 20 to 40 years old were offered by the Department of Anatomy of Sun Yat-sen University. Anteroposterior diameters of the pelvic inlet (the distance from midpoint of superior margin of pubic symphysis to midpoint of superior margin of promontory) and transverse diameters of the pelvic inlet (the maximum distance between left and right iliac crest) were measured. Eight pelvic specimens were selected because their anteroposterior diameters were $10.5 \mathrm{~cm} \sim 11.6 \mathrm{~cm}$ and the transverse diameters were $12.6 \mathrm{~cm} \sim 13.8 \mathrm{~cm}$ which showed these eight specimens were within a $5 \%$ of variation in pelvic bone size. The fifth lumbar vertebrae, the hip joint capsule and the proximal and middle portion of femur in every specimen were kept. Abnormalities, tumors and damages of pelvis were excluded by general observation and X-ray examination.

\section{Methods}

\section{Measurement of normal specimens}

After rejecting adherent soft tissue all pelvic specimens were entrapped within the homemade fixture with denture acrylic (Shanghai medical apparatus and instruments Ltd, China). The anterior superior iliac spine and the pubic symphysis plane of each specimen were kept in the same coronary and femurs were held in $15^{\circ}$ adduction angle in order to simulate human standing posture [22] (Figure 1a). Hip joint capsule was incised from lateral, and femoral head was cleaned up after it was dislocated so that the pressure sensitive paper (prescale low pressure (LW): 2.5-10Mpa, Fujifilm, Japan), which was cut according to the shape of each femoral head, could completely cover it [23]. Following that, femoral head was reduced and the specimen along with the fixture was installed on the material testing machine (SANS CMT6104, MTS Company, USA). Then, a hydraulic load at a speed of $5 \mathrm{~mm} / \mathrm{min}$ was applied on the preload of $200 \mathrm{~N}$ to detect the maximum stress region of femoral head [24]. The region was positioned according to the corresponding area where the discoloration was most obvious on the pressure sensitive paper which was removed from femoral head gently (Figure 1b). Then as we reported previously [24], the center of the maximum stress region of femoral head was marked for a strain gauge (KFG-5-120-C1-11L3M2R, sensitivity coefficient: $2.08 \pm 1.0 \%$, KYOWA Company, Japan) in biaxial. The shape of the strain gauge, $15 \mathrm{~mm} \times$ $5 \mathrm{~mm}$ in size and $33 \mu \mathrm{m} \sim 38 \mu \mathrm{m}$ in thickness, was adjusted with dimensions base on the curve of femoral head. It was pasted onto articular cartilage at the marked central region of the maximum stress area using quick-drying adhesive (cyanoacrylate adhesive CC-33A, KYOWA Company, Japan) without any bubble. And it could cover $34 \%$ of the half sphere in the mediolateral direction of femoral head. Then an accessory film of polyethylene resin from KYOWA was used to cover the strain gauge. A latex cover was placed over the strain gauge, and waited for about 60 minutes until the adhesive was completely harden. Afterward the specimen was fixed in the material testing machine again and the hip strain value was measured with
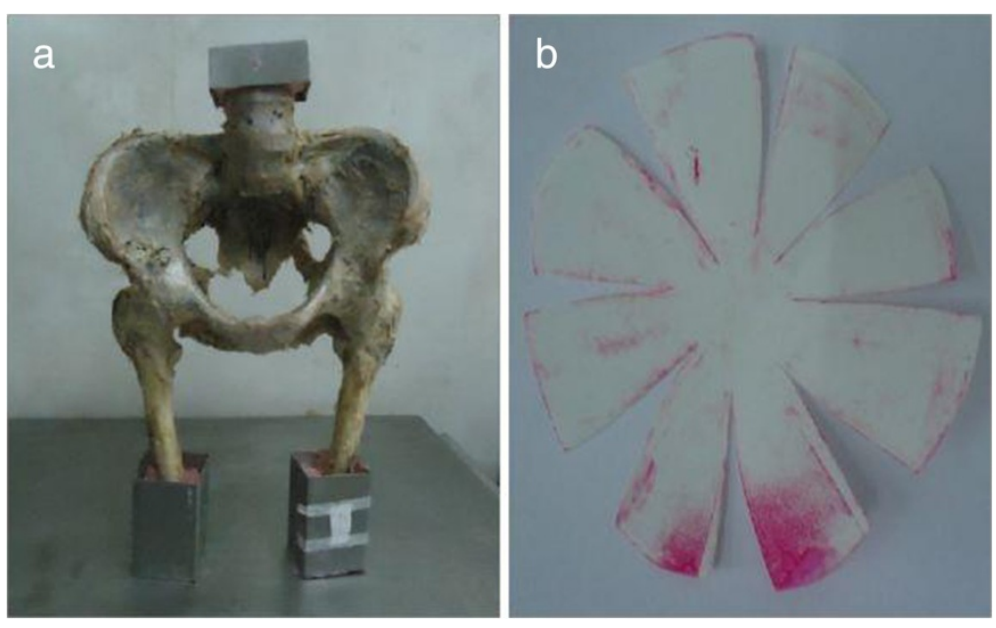

Figure 1 Fixation of pelvic specimen and color change of the Fuji film. a. the longitudinal axis of femoral diaphysis and median line of pelvis was in $15^{\circ}$ adduction angle. $\mathbf{b}$. the area of Fuji film where color change was most obvious. 
KYOWA PCD-2300A strain data analysis system (KYOWA Company, Japan) when a hydraulic load was applied at a speed of $5 \mathrm{~mm} / \mathrm{min}$ until $600 \mathrm{~N}$ [25]. Every hip was measured three times.

\section{Establishment and measurement of DDH model}

$\mathrm{X}$-ray of pelvis was taken for each specimen and Wiberg central-edge angle (CE angle) was measured on the X-ray film. Firstly, a homemade metallic scale with $2 \mathrm{~mm}$ accuracy of measurement was fixed just above the acetabulum of the specimen using two Kirschner wires. X-ray was took repeatedly to ensure the lower edge of the scale was parallel to the connecting line between bilateral acetabular upper edges, so that it could be used as the reference when making a dysplastic hip (Figure 2c). Then we drew a predicted $1.0 \mathrm{~cm}$ bone-cut line on the posterior and superior border of acetabulum (Figure 2a). After resecting the bone with a chisel [26] (Figure 2b), the pelvis X-ray film was retaken to affirm that the $\mathrm{CE}$ angle of the build hip was 10 to 20 degrees (Figure 2d). So our model was consistent with DDH in morphology and imaging examination. Then the maximum stress region of femoral head of the DDH model was located using the method mentioned above. Similarly, the DDH model was fastened on the material testing machine and hip strain value was measured three times in the aforementioned way.

\section{Performance of three osteotomies and measurement}

After establishment and measurement of DDH model, shelf procedure, Chiari osteotomy and RAO were performed on the specimens by the same surgeon (S.X.). The shelf procedure was carried out on both hip joints of each model and the bone fragments of width $1.0 \mathrm{~cm}$ were used (Figure 3a). After removing the bone fragments of shelf procedure, Chiari osteotomy was performed on the right hip of each specimen and RAO was performed on the left hip (Figure 3b and c). For Chiari osteotomy, because the maximal movement distance was $1.0 \mathrm{~cm}$ due to its own limitation of antiseptic cadaver specimen, the bone fragment was tried to be moved inward by $1.0 \mathrm{~cm}$. At the same time, the bone fragment was rotated outward by $1.0 \mathrm{~cm}$ in RAO. X-ray of pelvis was taken and $C E$ angles were confirmed in the normal range after every procedure (Figure $3 \mathrm{e}-\mathrm{f}$ ). The pressure sensitive paper was used to detect the maximum stress region of femoral head after each osteotomy and hip strain value of the detected region was obtained as described above.

\section{Statistical analysis}

All data were presented as mean and standard deviation. We assumed that hip strain value of the DDH model before osteotomy was $\alpha$ and the value after osteotomy was $\beta$, and then the improved ratio of osteotomy of hip strain of DDH model was equal to $(\alpha-\beta) / \alpha$. The differences of hip strain values before and after osteotomy within the same group were compared with pairedsample $T$ test. One-way analysis of variance was applied in comparison among these three groups and SNK test was adopted in comparison between any two groups. All
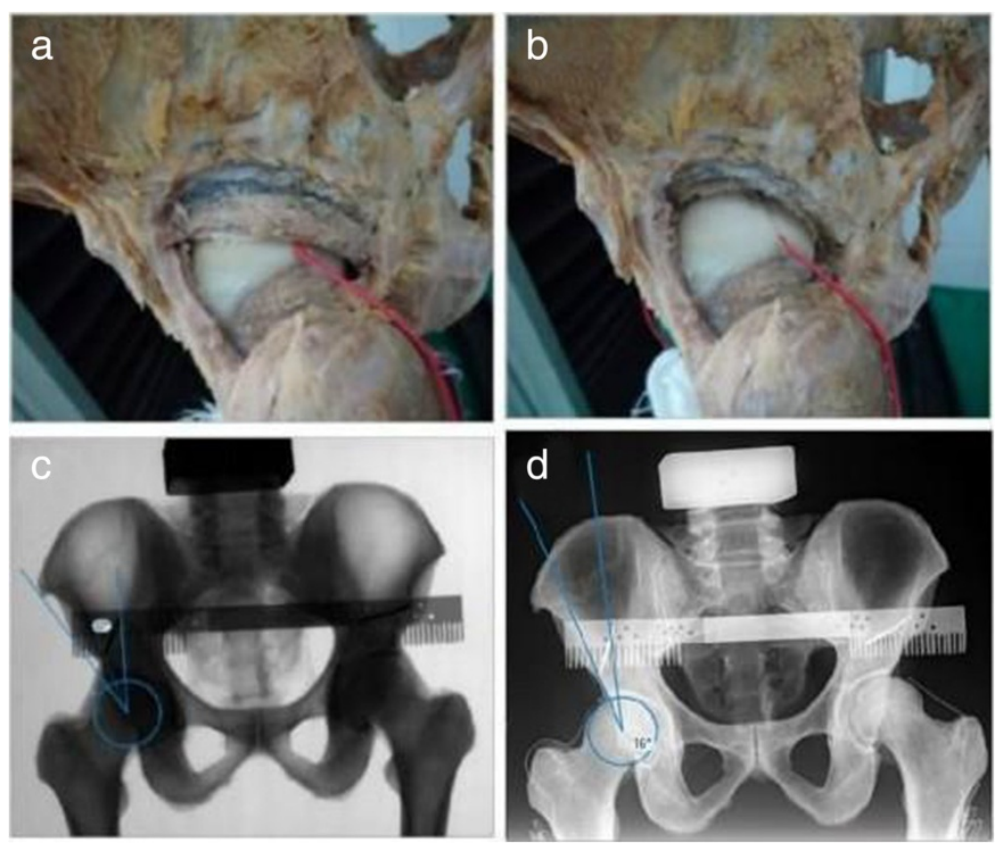

Figure $\mathbf{2}$ The model of DDH. $\mathbf{a}$ and $\mathbf{b}$ were the appearance of specimen before and after osteotomy; $\mathbf{c}$ and $\mathbf{d}$ were the $\mathrm{X}$-ray pictures before and after osteotomy, and the CE angle was $32^{\circ}$ before osteotomy, and it turned to $16^{\circ}$ after osteotomy. 


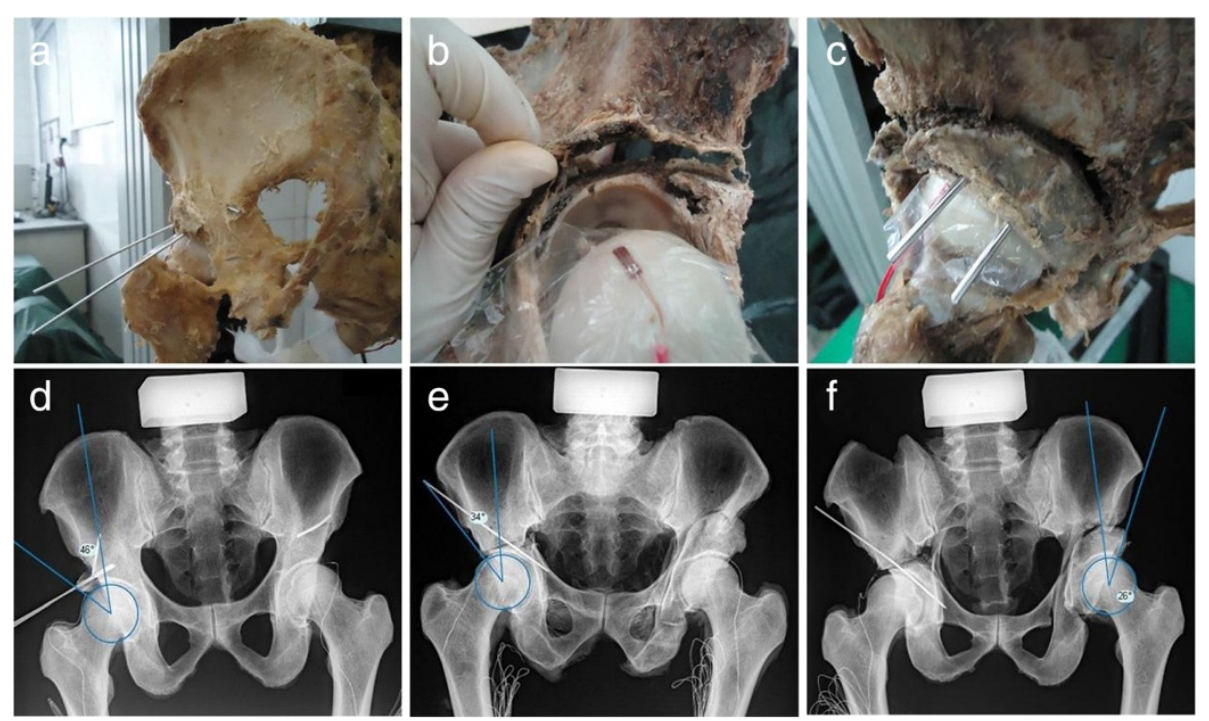

Figure 3 The three different treatment options for DDH. a-c were the appearance of specimen after shelf procedure (a), Chiari osteotomy (b) and RAO (c); d-f were the X-ray pictures after shelf procedure (d), Chiari osteotomy (e) and RAO (f), and CE angles after every osteotomy were all greater than 20 degrees.

statistical analyses were performed using SPSS version 13.0 and $P$ values $<0.05$ were considered statistically significant.

\section{Results}

The maximum stress region of femoral head was consistent in all normal pelvis specimens, all of which were located on the site corresponding to the posterior border of acetabulum. Comparing with normal specimens, the maximum stress region of DDH model was shifted outward the site where the femoral head contacted with posterior border of acetabulum because $\mathrm{CE}$ angle became smaller.

The strain values of left and right hip in normal specimens were 646.88 (SD 789.98) and 955.94 (SD 837.42) respectively, while they were obviously increased about two times in DDH models to 1439.03 (SD 625.23) and 1558.75 (SD 1009.46) (Tables 1 and 2). According to the morphology, imaging examination and these biomechanical results, the DDH model was considered to be successful.

After shelf procedure, the strain value of left hip was decreased to 1083.13 (SD 784.51) and that of right hip was dropped to 816.88 (SD 671.03), both of which had significant differences before and after the procedure
$(\mathrm{P} 1$ eft $=0.016$ and Pright $=0.021)$. Likewise, the strain value of left hip was significantly declined to 574.94 (SD 430.88) after RAO $(\mathrm{P}=0.004)$. However, the average strain value of right hip was raised to 1614.81 (SD 932.67) after the Chiari osteotomy because five of these hips' strain values were increased by $268.00 \sim 1149.50$, averaged 622.70. Nevertheless, no significant difference of hip strain value was found before and after Chiari osteotomy $(\mathrm{P}=0.856)$. Moreover, the improved ratio of RAO treatment was better than shelf procedure significantly $(P=0.015)$ and Chiari osteotomy $(P=0.0007)$, and the descendent range of shelf procedure was greater than Chiari osteotomy significantly $(\mathrm{P}=0.018)$ (Figure 4$)$.

\section{Discussion}

DDH patients with acetabular and femoral head developmental defect often have an abnormal shape and/or altered position of acetabulum, which leads to insufficient covering of femoral head or mismatch of femoral head and acetabulum. Consequently, the anatomical basis that maintains hip biomechanical stability changes thereby makes the femoral head prone to lateral displacement and secondary subluxation. These changes in anatomical structure result in reduction of loading area of hip and obvious increase of the tension, pressure and

Table 1 The relative strain values of normal specimens before and after establishment of DDH (left) (10-6)

\begin{tabular}{cccccccccc}
\hline NO. & A & B & C & D & E & F & G & H & Average \\
\hline Before & 720.00 & 370.00 & 1912.50 & -795.00 & 1027.50 & 1125.00 & 650.00 & 165.00 & 646.88 \\
After & 1126.25 & 940.00 & 2386.00 & 932.50 & 1245.00 & 2420.00 & 1505.00 & 957.50 & 1439.03 \\
After/Before & 1.56 & 2.54 & 1.25 & 1.17 & 1.21 & 2.15 & 2.32 & 5.80 & 2.25 \\
\hline
\end{tabular}


Table 2 The relative strain values of normal specimens before and after establishment of DDH (right) (10-6)

\begin{tabular}{cccccccccc}
\hline NO. & A & B & C & D & E & F & G & H & Average \\
\hline Before & 957.50 & 225.00 & 842.50 & 952.50 & 2900.00 & 700.00 & 250.00 & 820.00 & 955.94 \\
After & 1005.00 & 540.00 & 2235.00 & 1175.00 & 3395.00 & 905.00 & 735.00 & 2430.00 & 1552.50 \\
After/Before & 1.05 & 2.40 & 2.65 & 1.23 & 1.17 & 1.29 & 2.94 & 2.96 & 1.96 \\
\hline
\end{tabular}

shear stress of articular surface. Comparing with normal hip, hip of DDH has a $26 \%$ reduction of the contact area and a $23 \%$ increment of pressure [27]. Osteoarthritis occurs when the pressure exceeds the allowable range of osteoblasts and chondrocytes. Rotational acetabular osteotomy, Chiari osteotomy and shelf procedure are three effective treatment options for $\mathrm{DDH}$, which could delay the onset of secondary osteoarthritis. Here, we identified the trend of biomechanical change on these three acetabular osteotomies, through establishing sixteen DDH models of human cadaver specimens.

The shelf procedure could alleviate the hip pressure and reduce the tension of the capsule without causing hip stiffness postoperative. And this operation is relatively simple and safe without disruption of the pelvic ring structure. Hence, it can be used for young patients with subluxation of hip, providing good stability and mobility, postponing subsequent arthroplasty. Moreover, because it merely affects the hip structure, it would not increase the difficulty of the arthroplasty. However, the procedure has the risk of bone resorption and collapse. After a follow-up of 140 patients 157 hips for 5 years, Staheli found that $83.3 \%$ of the patients had a satisfactory hip function recovery after the shelf procedure. In our experiment [28], we found that the shelf procedure could obviously reduce hip stress of DDH.

In a follow-up study of $96 \mathrm{DDH}$ patients 100 hips after Chiari osteotomy, Matsuno found that $84 \%$ patients' clinical symptoms improved postoperatively [29]. Chiari osteotomy is intended to move the hip inward from osteotomy line in order to increase the bony coverage of femoral head. However, the increased coverage does not match the shape of femoral head. Our results showed no significant change in hip stress before and after Chiari osteotomy. Even five of these specimens had an increased hip stress postoperatively. One possible reason for this outcome is that when the hip was moved inward, femoral head became closer to the center of gravity line which changed the conducting direction of weightbearing force and raised the pressure of hip. Whereas our experiment was a biomechanical study within a short period after osteotomy, it could not reveal the long-term effect of Chiari osteotomy. In clinic, the capsule could turn to metaplastic fibrocartilage and then form a new acetabulum over long term, which could expand the contact area of femoral head and acetabulum and distribution of hip stress. Consequently, function of hip was improved and the occurrence of osteoarthritis was delayed. It had been reported that as a result of the establishment of a larger contact zone, the contact pressure distribution of the hip was close to normal two years after Chiari osteotomy in a photoelastic study [20]. Even so, whether biomechanical property of hip could be improved in vivo when the metaplastic fibrocartilage becomes a part of acetabulum still remains to be further studied.

After RAO, the position relation between acetabulum and femoral head is readjusted to increase acetabular coverage of femoral head and reduce acetabular shear force that lead to restoration of normal hip joint anatomy and biomechanical properties. Previous studies have reported that RAO could obviously improve clinical symptoms and hip function of $\mathrm{DDH}$ patients and achieve satisfactory clinical outcomes [30]. Our data suggested that RAO could greatly reduce hip stress of the $\mathrm{DDH}$ model due to congruence between acetabulum and femoral head postoperatively, which was better than shelf procedure and Chiari osteotomy. From another

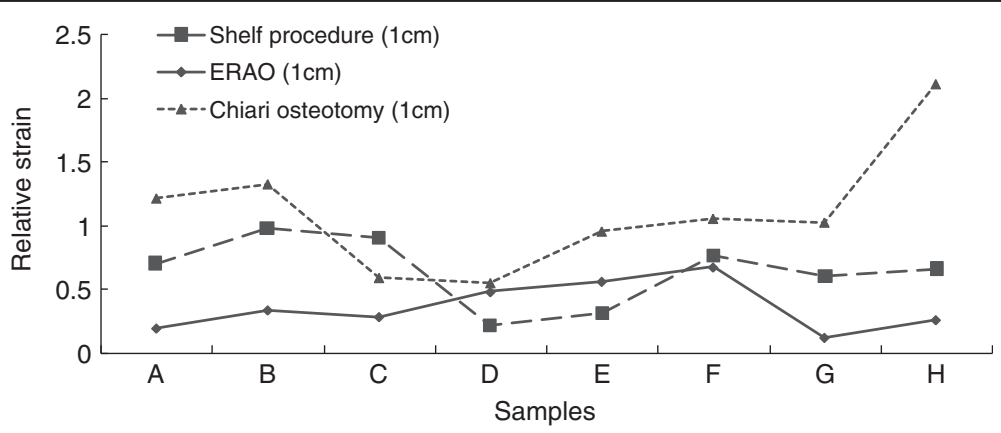

Figure 4 Strain trend of femoral heads in different surgical osteotomies for DDH models. 
aspect, unlike the long term articular cartilage after Chiari osteotomy which consists of fibrocartilage transformed from joint capsule, the articular cartilage after RAO is the own hyaline cartilage of the hip with better tissue compatibility so that RAO could greatly delay the progression of osteoarthritis. It was reported that about $80 \%$ patients who underwent this operation avoided total hip replacement in the future [31]. While similar studies of Chiari osteotomy and shelf procedure were so far lacking. Therefore, we believe that for young DDH patients who need surgical interventions but not to the point at which the arthroplasty is required, RAO may be a better choice with greater advantages.

However, there are some limitations in this research. First of all, this is a measurement study base on cadaver specimens, and thus the data had certain difference from real human. Next, the biomechanical characteristic of human body is so complicated that it cannot be real simulated completely in vitro. And strain measurement at only one time-point is insufficient because biological effects are always different at various points in time. In addition, there was only one strain point in this research, but this point was located in the maximum stress region of femoral head, so it was able to reflect the force condition of hip to some extent. Certainly, we will also design more strain points to better reflect the force condition in further study. As we focused on the hip stress when standing, the changes of hip stress in different directions after three kinds of surgical methods require further exploration combined with finite element analysis and detection of muscle force. Furthermore, the ideal strain gage should change resistance only due to the deformations of the surface to which the sensor is attached. However, in real applications, temperature, material properties, the adhesive that bonds the gage to the surface, and the stability of the metal all will affect the detected resistance. Therefore, all of these limitations should be corrected in our future research.

\section{Conclusions}

In summary, from our findings, RAO and shelf procedure, especially the former are effective treatments for DDH in the biomechanics.

\section{Competing interests}

The authors declare that they have no competing interests.

\section{Authors' contributions}

M.F. and S.X. carried out the majority of the experiments, analyzed data and prepared the manuscript; Z.Z. and G.H. assisted with the experiments and the analysis of the data; J.L., X.D., Z.Y., P.W. and W.L. provided suggestions for the study and critically reviewed the manuscript; M.F. and Z.Z. supervised the project and wrote most of the manuscript. All authors read and approved the final manuscript.

\section{Acknowledgements}

We are grateful to Tyler Smallman from Washington University School of Medicine for the critical reading of this paper. This work was funded by China Science and Technology Grant of Guangdong (2007B031002003, 2009A030200013 and 2011B031300012) and Natural Science Foundation of China (81171759, 81201388).

\section{Author details}

'Department of Joint Surgery, First Affiliated Hospital, Sun Yat-sen University, Guangzhou 510080, China. 'Department of Orthopaedic Surgery, the Affiliated Jiangmen Hospital of Sun Yat-sen University, Jiangmen 529070, China.

Received: 2 September 2013 Accepted: 12 February 2014

Published: 21 February 2014

\section{References}

1. Aronson J: Osteoarthritis of the young adult hip: etiology and treatment. Instr Course Lect 1986, 35:119-128.

2. Solomon L, Schnitzler CM: Pathogenetic types of coxarthrosis and implications for treatment. Arch Orthop Trauma Surg 1983, 101(4):259-261.

3. Bombelli R, Santore RF, Poss R: Mechanics of the normal and osteoarthritic hip. A new perspective. Clin Orthop Relat Res 1984, 182:69-78.

4. Millis MB, Murphy SB, Poss R: Osteotomies about the hip for the prevention and treatment of osteoarthrosis. Instr Course Lect 1996, 45:209-226.

5. Hartofilakidis G, Karachalios T, Stamos KG: Epidemiology, demographics, and natural history of congenital hip disease in adults. Orthopedics 2000, 23(8):823-827.

6. Aldinger PR, Thomsen M, Mau H, Ewerbeck V, Breusch SJ: Cementless Spotorno tapered titanium stems: excellent 10-15-year survival in 141 young patients. Acta Orthop Scand 2003, 74(3):253-258.

7. Hartofilakidis G, Karachalios T, Zacharakis N: Charnley low friction arthroplasty in young patients with osteoarthritis. A 12- to 24-year clinical and radiographic followup study of 84 cases. Clin Orthop Relat Res 1997, 341:51-54.

8. Nakamura T, Yamaura M, Nakamitu S, Suzuki K: The displacement of the femoral head by rotational acetabular osteotomy. A radiographic study of 97 subluxated hips. Acta Orthop Scand 1992, 63(1):33-36.

9. Ninomiya S: Rotational acetabular osteotomy for the severely dysplastic hip in the adolescent and adult. Clin Orthop Relat Res 1989, 247:127-137.

10. Schramm M, Pitto RP, Rohm E, Hohmann D: Long-term results of spherical acetabular osteotomy. J Bone Joint Surg (Br) 1999, 81(1):60-66.

11. Yasunaga $Y$, Ochi M, Shimogaki K, Yamamoto S, Iwamori H: Rotational acetabular osteotomy for hip dysplasia: 61 hips followed for 8-15 years. Acta Orthop Scand 2004, 75(1):10-15.

12. Yasunaga $Y$, Takahashi $K$, Ochi M, Ikuta $Y$, Hisatome $T$, Nakashiro J, Yamamoto S: Rotational acetabular osteotomy in patients forty-six years of age or older: comparison with younger patients. J Bone Joint Surg Am 2003, 85-A(2):266-272

13. Hamanishi C, Tanaka S, Yamamuro T: The Spitzy shelf operation for the dysplastic hip. Retrospective 10 (5-25) year study of 124 cases. Acta Orthop Scand 1992, 63(3):273-277.

14. Kerboull M: General therapeutic indications in congenital hip dislocation in adults. Acta Orthop Belg 1990, 56(1 Pt B):407-413.

15. Colton $\mathrm{CL}$ : Chiari osteotomy for acetabular dysplasia in young subjects. $J$ Bone Joint Surg (Br) 1972, 54(4):578-589.

16. Karami M, Fitoussi F, llharreborde B, Pennecot GF, Mazda K, Bensahel H: The results of Chiari pelvic osteotomy in adolescents with a brief literature review. J Child Orthop 2008, 2(1):63-68.

17. Mitchell GP: Chiari medial displacement osteotomy. Clin Orthop Relat Res 1974, 98:146-150.

18. Rozkydal Z, Kovanda M: Chiari pelvic osteotomy in the management of developmental hip dysplasia: a long term follow-up. Bratis/ Lek Listy 2003, 104(1):7-13.

19. Teuffer AP, Noguera JG: Experience with innominate osteotomy (Salter) and medial displacement osteotomy (Chiari) in the treatment of acetabular dysplasia. Preliminary report of 82 operations. Clin Orthop Relat Res 1974, 98:133-136.

20. Iliescu N, Pastrama SD, Gruionu LG, Jiga G: Biomechanical changes of hip joint following different types of corrective osteotomy-photoelastic studies. Acta Bioeng Biomech 2008, 10(3):65-71. 
21. Karami M, Gouran SD, Ghadirpoor A, Rahimpour S, Azghani M, Farahmand F: A computer model for evaluating the osteotomy parameters of Chiari pelvic osteotomy. Int Orthop 2010, 34(3):329-333.

22. Bergmann G, Deuretzbacher G, Heller M, Graichen F, Rohlmann A, Strauss J, Duda GN: Hip contact forces and gait patterns from routine activities. J Biomech 2001, 34(7):859-871.

23. Michaeli DA, Murphy SB, Hipp JA: Comparison of predicted and measured contact pressures in normal and dysplastic hips. Med Eng Phys 1997, 19(2):180-186.

24. Zhang Z, Fu M, Kang Y, Chen Y, Liao W: Upward and inward displacements of the acetabular component increase stress on femoral head in single endoprothesis models. Int Orthop 2010, 34(4):479-483.

25. Ostbyhaug PO, Klaksvik J, Romundstad P, Aamodt A: An in vitro study of the strain distribution in human femora with anatomical and customised femoral stems. J Bone Joint Surg (Br) 2009, 91(5):676-682.

26. Giori NJ, Trousdale RT: Acetabular retroversion is associated with osteoarthritis of the hip. Clin Orthop Relat Res 2003, 417:263-269.

27. Hipp JA, Sugano N, Millis MB, Murphy SB: Planning acetabular redirection osteotomies based on joint contact pressures. Clin Orthop Relat Res 1999, 364:134-143.

28. Staheli LT, Chew DE: Slotted acetabular augmentation in childhood and adolescence. J Pediatr Orthop 1992, 12(5):569-580.

29. Migaud H, Chantelot C, Giraud F, Fontaine C, Duquennoy A: Long-term survivorship of hip shelf arthroplasty and Chiari osteotomy in adults. Clin Orthop Relat Res 2004, 418:81-86.

30. Maruyama M, Wakabayashi S, Tensho K: Less invasive rotational acetabular osteotomy for hip dysplasia. Clin Orthop Relat Res 2013, 471(4):1263-1270.

31. Shimogaki K, Yasunaga Y, Ochi M: A histological study of articular cartilage after rotational acetabular osteotomy for hip dysplasia. J Bone Joint Surg (Br) 2005, 87(7):1019-1023.

doi:10.1186/1471-2474-15-47

Cite this article as: Fu et al.: The biomechanical differences of rotational acetabular osteotomy, Chiari osteotomy and shelf procedure in developmental dysplasia of hip. BMC Musculoskeletal Disorders 2014 15:47.

\section{Submit your next manuscript to BioMed Central and take full advantage of:}

- Convenient online submission

- Thorough peer review

- No space constraints or color figure charges

- Immediate publication on acceptance

- Inclusion in PubMed, CAS, Scopus and Google Scholar

- Research which is freely available for redistribution 